Math. Model. Nat. Phenom.

Vol. 5, No. 6, 2010, pp. 180-195

DOI: $10.1051 / \mathrm{mmnp} / 20105609$

\title{
An Optimal Control Problem for a Predator-Prey Reaction-Diffusion System
}

\author{
N. C. Apreutesei * \\ Department of Mathematics, Technical University "Gh. Asachi” Iasi, 11, Bd. Carol I \\ 700506 Iasi, Romania
}

\begin{abstract}
An optimal control problem is studied for a predator-prey system of PDE, with a logistic growth rate of the prey and a general functional response of the predator. The control function has two components. The purpose is to maximize a mean density of the two species in their habitat. The existence of the optimal solution is analyzed and some necessary optimality conditions are established. The form of the optimal control is found in some particular cases.
\end{abstract}

Key words: adjoint system, functional response of the predator, logistic growth rate, maximum principle, optimality conditions

AMS subject classification: 49K20, 92D25, 93C10, 93C15, 35K50, 35K55

\section{Introduction}

We study an optimal control problem related to the predator-prey system

$$
\left\{\begin{array}{c}
\frac{\partial y_{1}}{\partial t}=\alpha_{1} \Delta y_{1}+r y_{1}\left(1-\frac{y_{1}}{k}\right)-y_{2} F\left(y_{1}, y_{2}\right) \\
\frac{\partial y_{2}}{\partial t}=\alpha_{2} \Delta y_{2}+y_{2}\left[-d+c F\left(y_{1}, y_{2}\right)\right]
\end{array},(t, x) \in Q=[0, T] \times \Omega\right.
$$

where $\Omega$ is an open bounded set from $R^{m}, m \leq 3$, with the boundary $\partial \Omega$ sufficiently smooth. Here $y_{1}(t, x)$ and $y_{2}(t, x)$ represent the densities of prey and predator species, respectively, at the moment $t \in[0, T]$ and the spatial position $x \in \Omega$ and $\alpha_{1}, \alpha_{2}, r, k, c, d>0$ are given parameters.

*E-mail: napreut@gmail.com 
Function $f\left(y_{1}\right)=r y_{1}\left(1-\frac{y_{1}}{k}\right)$ is the logistic growth rate of the prey species. The parameter $r$ controls the prey population growth, while the coefficient $k$ is the prey-carrying capacity. The predator functional response $F\left(y_{1}, y_{2}\right)$ signifies the density of prey consumed per predator in unit time. It is supposed to satisfy the following conditions:

(i) $F\left(y_{1}, y_{2}\right)=y_{1} \widetilde{F}\left(y_{1}, y_{2}\right)$, with $\widetilde{F} \in C^{1}\left((0, \infty)^{2}\right)$;

(ii) $\widetilde{F}$ and its first partial derivatives are bounded on bounded sets;

(iii) $F\left(y_{1}, y_{2}\right)>0, \frac{\partial F}{\partial y_{1}}\left(y_{1}, y_{2}\right)>0$ and $F\left(y_{1}, y_{2}\right)+y_{2} \frac{\partial F}{\partial y_{2}}\left(y_{1}, y_{2}\right)>0$, for all $y_{1}, y_{2}>0$.

Function $F$ includes as particular cases various classical functional responses ([9], [10]):

$F\left(y_{1}, y_{2}\right)=b y_{1}$ (Holling type I), $F\left(y_{1}, y_{2}\right)=\frac{b y_{1}}{1+a y_{1}}$ (Holling type II), $F\left(y_{1}, y_{2}\right)=\frac{b y_{1}^{2}}{1+a y_{1}^{2}}$ (Holling type III), $F\left(y_{1}, y_{2}\right)=\frac{b y_{1}^{n}}{y_{2}^{\gamma}+a y_{1}^{n}}$ (Hassell \& Valery), $F\left(y_{1}, y_{2}\right)=\frac{b y_{1}}{y_{1}+a y_{2}+a y_{0}}(\mathrm{De}$ Angelis et al; Beddington), etc. The parameters $a, b, n, \gamma$ are positive. All these functional responses satisfy conditions $(i)-($ iii $)$, the penultimate one for $0<\gamma \leq 1$ and $n \geq 1$.

The predator's numerical response $G\left(y_{1}, y_{2}\right)=-d+c F\left(y_{1}, y_{2}\right)$ shows the per capita growth rate of the predator population. Parameters $d$ and $c$ are the per capita predator death rate and the maximal per capita predator birth rate, respectively. More details about these models and their significance can be found in [9] and [10].

One separates the prey from the predators with the aid of a control function $u: Q \rightarrow R$, $0 \leq u(t, x) \leq 1$ a. e. on $Q$. Then the term $y_{2} F\left(y_{1}, y_{2}\right)$ from the system is multiplied by $u$. The mixture rate at the moment $t$ and point $x$ is $u(t, x)$. We also introduce a control function $v: Q \rightarrow R, 0<a \leq v(t, x) \leq 1$ a. e. on $Q$, which signifies the rate of mixture between the individuals of the prey population. Then the second term of the prey's logistic growth rate is multiplied by $v$. Function $v$ is here strictly positive. This means that the prey individuals can not be completely separated from each other. The dynamics of the controlled ecosystem is given by

$$
\left\{\begin{array}{c}
\frac{\partial y_{1}}{\partial t}=\alpha_{1} \Delta y_{1}+r y_{1}\left(1-\frac{y_{1}}{k} v\right)-u y_{2} F\left(y_{1}, y_{2}\right) \\
\frac{\partial y_{2}}{\partial t}=\alpha_{2} \Delta y_{2}+y_{2}\left[-d+c u F\left(y_{1}, y_{2}\right)\right]
\end{array}\right.
$$

for $(t, x) \in[0, T] \times \Omega=Q$. One associates homogeneous Neumann boundary conditions

$$
\frac{\partial y_{1}}{\partial \nu}=\frac{\partial y_{2}}{\partial \nu}=0, \quad(t, x) \in(0, T) \times \partial \Omega=\Sigma
$$

(i. e. we have an isolated environment) and initial conditions of the form

$$
y_{1}(0, x)=y_{1}^{0}(x)>0, y_{2}(0, x)=y_{2}^{0}(x)>0, x \in \Omega .
$$

Our goal is to find the optimal control $(u, v)$ such that the total density of the two species to be maximized. More exactly, we want to minimize the cost functional

$$
J(u, v)=-\int_{\Omega}\left[l_{1} y_{1}(T, x)+l_{2} y_{2}(T, x)\right] d x-\int_{0}^{T} \int_{\Omega}\left[k_{1} y_{1}(t, x)+k_{2} y_{2}(t, x)\right] d t d x,
$$


where the constants $l_{1}, l_{2}, k_{1}, k_{2} \geq 0$ are not all zero, $\left(y_{1}, y_{2}\right)$ is the solution to $(1.1)-(1.3)$ and $(u, v)$ belongs to the admissible set

$$
\mathcal{U}=\left\{(u, v) \in\left(L^{2}(Q)\right)^{2}, 0 \leq u(t, x) \leq 1,0<a \leq v(t, x) \leq 1 \text { a. e. on } Q\right\} .
$$

If $k_{1}=k_{2}=0$ and $l_{1}=l_{2}=1$, the problem is to maximize the total density of the two populations in the end of the time interval [0,T]. Similar problems for ODE were analyzed in [1], [2], [13]. Some optimal control problems for related reaction-diffusion equations were investigated in [5], [7]. For general mathematical methods in the theory of optimal control, the reader may refer to [3].

Papers [4] and [6] are concerned with the optimality conditions for some control problems of age-structured population models, while maximum principle for linear size-structured population systems is obtained in [8].

In this paper we first study the existence and uniqueness of the global solution to problem $(1.1)-(1.3)$. One shows that a strong solution exists and it is positive and bounded on the whole set $Q$ (Section 2). Other existence results on the global solutions for predator-prey systems are studied in [12]. In Section 3 we prove the existence of an optimal solution to problem (1.1)-(1.4). First order necessary conditions of optimality are established in Section 4. We find the form of $u$ and $v$ and show that $v$ is constant on $Q$, namely $v=a$. Finally, we deduce that in some particular cases $u$ is zero.

\section{The existence of the global solution to the boundary value problem}

In this section we study the existence of a strong solution for problem (1.1) - (1.3) and show that it is positive and bounded. We work in the Hilbert space $H=L^{2}(\Omega)^{2}$. To this end, denote by $A$ the operator $A: D(A) \subset L^{2}(\Omega)^{2} \rightarrow L^{2}(\Omega)^{2}$,

$$
A\left(\begin{array}{l}
y_{1} \\
y_{2}
\end{array}\right)=\left(\begin{array}{c}
\alpha_{1} \Delta y_{1} \\
\alpha_{2} \Delta y_{2}
\end{array}\right)
$$

with the domain

$$
D(A)=\left\{y=\left(\begin{array}{l}
y_{1} \\
y_{2}
\end{array}\right) \in H^{2}(\Omega)^{2}, \frac{\partial y_{1}}{\partial \nu}=\frac{\partial y_{2}}{\partial \nu}=0 \text { a. e. on } \partial \Omega\right\},
$$

and by $g:[0, T] \times \Omega \times R \rightarrow R$ the nonlinear term from the system (1.1),

$$
g(t, x, y)=\left(\begin{array}{c}
g^{1}(t, x, y) \\
g^{2}(t, x, y)
\end{array}\right)=\left(\begin{array}{c}
r y_{1}\left(1-\frac{y_{1}}{k} v\right)-u y_{2} F\left(y_{1}, y_{2}\right) \\
y_{2}\left[-d+c u F\left(y_{1}, y_{2}\right)\right]
\end{array}\right)
$$


for $y=\left(y_{1}, y_{2}\right)^{T}$ and $u=u(t, x), v=v(t, x)$. Here and everywhere below the superscript ${ }^{T}$ from $\alpha^{T}$ means the transposed of $\alpha$. Now let $f:[0, T] \times D(f) \rightarrow L^{2}(\Omega)^{2}$ be given by $f=\left(f^{1}, f^{2}\right)^{T}$,

$$
\begin{gathered}
D(f)=\left\{y \in L^{2}(\Omega)^{2}, g(t, \cdot, y(\cdot)) \in L^{2}(\Omega)^{2},(\forall) t \in[0, T]\right\}, \\
f(t, y)(x)=\left(f^{1}(t, y), f^{2}(t, y)\right)(x)=g(t, x, y(x)),
\end{gathered}
$$

for all $y=\left(y_{1}, y_{2}\right)^{T} \in D(f)$, all $t \in[0, T]$, and a. e. $x \in \Omega$. Assume that $y^{0}=\left(y_{1}^{0}, y_{2}^{0}\right)^{T} \in$ $D(A)$. Then, problem $(1.1)-(1.3)$ can be expressed as an abstract Cauchy problem in the real Hilbert space $H=L^{2}(\Omega)^{2}$ :

$$
y^{\prime}(t)=A y(t)+f(t, y(t)), t \in[0, T], y(0)=y^{0} \in D(A) .
$$

To prove the existence for problem $(1.1)-(1.3)$, we will use the following result which can be found in [11], p. 189-190.

Theorem 1. Let $A$ be the infinitesimal generator of a $C_{0}$-semigroup of linear operators $T(t), t \geq$ 0 , on the reflexive Banach space $X$. If $f:[0, T] \times X \rightarrow X$ is Lipschitz continuous in $(t, y)$ on $[0, T] \times X$ and $y^{0} \in D(A)$, then problem (2.4) admits a unique (global) strong solution $y \in W^{1,2}(0, T ; X)$, with $y \in L^{2}(0, T ; D(A))$. If $f:[0, T] \times X \rightarrow X$ is only locally Lipschitz continuous on $X$, uniformly with respect to $t \in[0, T]$, then for every $y^{0} \in D(A)$, the initial value problem (2.4) has a strong solution y defined on a maximal interval $[0, \delta)$. Moreover, if $\delta<T$, then $\lim _{t / \delta}\|y(t)\|=\infty$.

Since in our case function $f$ is not Lipschitz continuous in $y$ uniformly with respect to $t$, we cannot use the above result directly. In fact, $f$ is not defined on the whole space $H$, but on $D(f)$. One associates the truncated initial value problem (see [7])

$$
y_{N}^{\prime}(t)=A y_{N}(t)+f^{N}\left(t, y_{N}(t)\right), t \in[0, T], y_{N}(0)=y^{0}
$$

where the positive number $N$ satisfies

$$
N>2 \max \left\{\left\|y_{1}^{0}\right\|_{L^{\infty}(\Omega)},\left\|y_{2}^{0}\right\|_{L^{\infty}(\Omega)}\right\}
$$

$y_{N}=\left(y_{1}^{N}, y_{2}^{N}\right)^{T}, f^{N}(t, y(t))=\left(f_{1}^{N}(t, y(t)), f_{2}^{N}(t, y(t))\right)^{T}$ and $f_{1}^{N}, f_{2}^{N}$ are defined as follows. If $y_{1}$ (or $y_{2}$ ) is greater than $N$ or less than $-N$, then $y_{1}$ (or $y_{2}$ ) from $f(t, y(t)$ ) is replaced by $N$ or by $-N$, respectively. In such a way, $f_{1}^{N}$ and $f_{2}^{N}$ are defined on $[0, T] \times H$ and become bounded on $Q$ and Lipschitz continuous in $y \in H$, uniformly with respect to $t \in[0, T]$. Since $y^{0} \in D(A)$, then making use of Theorem 1, we derive that problem (2.5) has a unique strong solution $y_{N} \in$ $W^{1,2}(0, T ; H) \cap L^{2}(0, T ; D(A))$. It can be also shown that $y_{N} \in L^{\infty}\left(0, T ; H^{1}(\Omega)^{2}\right)$.

We now prove that $y_{1}^{N}, y_{2}^{N} \in L^{\infty}(Q)$. To this end, denote

$$
K_{N}=\max \left\{\left\|f_{1}^{N}\right\|_{L^{\infty}(Q)},\left\|f_{2}^{N}\right\|_{L^{\infty}(Q)},\left\|y_{1}^{0}\right\|_{L^{\infty}(\Omega)},\left\|y_{2}^{0}\right\|_{L^{\infty}(\Omega)}\right\} .
$$


Then function $z_{1}^{N}(t, x)=y_{1}^{N}(t, x)-K_{N} t-\left\|y_{1}^{0}\right\|_{L^{\infty}(\Omega)}$ is the solution of the Cauchy problem

$$
\left\{\begin{array}{c}
\frac{d}{d t} z_{1}^{N}(t)=\alpha_{1} \Delta z_{1}^{N}(t)+f_{1}^{N}\left(t, y_{1}^{N}, y_{2}^{N}\right)-K_{N}, t \in[0, T] \\
z_{1}^{N}(0)=y_{1}^{0}-\left\|y_{1}^{0}\right\|_{L^{\infty}(\Omega)}
\end{array}\right.
$$

This problem admits a unique strong solution which is defined by

$$
z_{1}^{N}(t)=\widetilde{S}(t)\left(y_{1}^{0}-\left\|y_{1}^{0}\right\|_{L^{\infty}(\Omega)}\right)+\int_{0}^{t} S(t-s)\left(f_{1}^{N}\left(s, y_{1}^{N}, y_{2}^{N}\right)-K_{N}\right) d s
$$

where $\widetilde{S}(t)$ is the $C_{0}$-semigroup generated by $\alpha_{1} \Delta$. Since $y_{1}^{0}(x)-\left\|y_{1}^{0}\right\|_{L^{\infty}(\Omega)} \leq 0$ and $f_{1}^{N}-K_{N} \leq$ 0 , we get $z_{1}^{N}(t, x) \leq 0,(\forall)(t, x) \in Q$.

Similarly, denoting $w_{1}^{N}(t, x)=y_{1}^{N}(t, x)+K_{N} t+\left\|y_{1}^{0}\right\|_{L^{\infty}(\Omega)}$, we find that $w_{1}^{N}(t, x) \geq 0$, $(\forall)(t, x) \in Q$. These two inequalities imply that

$$
\left|y_{1}^{N}(t, x)\right| \leq K_{N} t+\left\|y_{1}^{0}\right\|_{L^{\infty}(\Omega)},(\forall)(t, x) \in Q,
$$

therefore $y_{1}^{N} \in L^{\infty}(Q)$. Analogously, $y_{2}^{N} \in L^{\infty}(Q)$, namely

$$
\left|y_{2}^{N}(t, x)\right| \leq K_{N} t+\left\|y_{2}^{0}\right\|_{L^{\infty}(\Omega)},(\forall)(t, x) \in Q .
$$

We prove now that $y_{1}^{N}>0, y_{2}^{N}>0$ on $Q$. The boundary value problem verified by $y_{1}^{N}$ is

$$
\left\{\begin{array}{c}
\frac{\partial y_{1}^{N}}{\partial t}=\alpha_{1} \Delta y_{1}^{N}+f_{1}^{N}\left(y_{1}^{N}, y_{2}^{N}\right),(t, x) \in Q \\
\frac{\partial y_{1}^{N}}{\partial \nu}=0,(t, x) \in \Sigma \\
y_{1}^{N}(0, x)=y_{1}^{0}(x), x \in \Omega .
\end{array}\right.
$$

Observe that $f_{1}^{N}$ is bounded on $Q$. We write $y_{1}^{N}=\left(y_{1}^{N}\right)^{+}-\left(y_{1}^{N}\right)^{-}$, where $\left(y_{1}^{N}\right)^{+}(t, x)=$ $\sup \left\{y_{1}^{N}(t, x), 0\right\}$ is the positive part of $y_{1}^{N}=y_{1}^{N}(t, x)$, while $\left(y_{1}^{N}\right)^{-}(t, x)=-\inf \left\{y_{1}^{N}(t, x), 0\right\}$ is the negative part of $y_{1}^{N}$. Multiplying (2.9) by $\left(y_{1}^{N}\right)^{-}$and using for $F$ the form in $(i)$, we deduce that

$$
\frac{1}{2} \frac{\partial}{\partial t}\left|\left(y_{1}^{N}\right)^{-}\right|^{2}=\alpha_{1}\left(y_{1}^{N}\right)^{-} \Delta\left(y_{1}^{N}\right)^{-}+\left|\left(y_{1}^{N}\right)^{-}\right|^{2}\left[r\left(1-\frac{y_{1}^{N}}{k} v\right)-u y_{2}^{N} \widetilde{F}\left(y_{1}^{N}, y_{2}^{N}\right)\right]
$$

Integrating over $\Omega$, via Green's formula and the boundary conditions for $y_{1}^{N}$, we get

$$
\begin{aligned}
& \frac{1}{2} \int_{\Omega} \frac{\partial}{\partial t}\left|\left(y_{1}^{N}\right)^{-}\right|^{2}(t, x) d x=-\alpha_{1} \int_{\Omega}\left|\nabla\left(y_{1}^{N}\right)^{-}\right|^{2}(t, x) d x+ \\
& +\int_{\Omega}\left|\left(y_{1}^{N}\right)^{-}\right|^{2}\left[r\left(1-\frac{y_{1}^{N}}{k} v\right)-u y_{2}^{N} \widetilde{F}\left(y_{1}^{N}, y_{2}^{N}\right)\right](t, x) d x .
\end{aligned}
$$


Integrating from 0 to $t, t \in[0, T]$ and making use of $(1.3),(2.7),(2.8)$, and of hypothesis $(i i)$, one arrives at

$$
\int_{\Omega}\left|\left(y_{1}^{N}\right)^{-}\right|^{2}(t, x) d x \leq C \int_{0}^{t} \int_{\Omega}\left|\left(y_{1}^{N}\right)^{-}\right|^{2}(s, x) d s d x,
$$

where $C>0$ is a constant depending only on $N$. By Gronwall's inequality, we obtain

$$
\int_{\Omega}\left|\left(y_{1}^{N}\right)^{-}\right|^{2}(t, x) d x \leq 0,
$$

so $y_{1}^{N}(t, x) \geq 0$ on $Q$. Similarly one obtains that $y_{2}^{N}(t, x) \geq 0$ on $Q$. Since $y_{i}^{0}(x)>0,(\forall) x \in \Omega$, $i=1,2$, we conclude that $y_{i}^{N}(t, x)>0,(\forall)(t, x) \in Q, i=1,2$.

We now choose $N$ satisfying (2.6). Then there exists $s \in(0, T)$ such that

$$
K_{N} s+\left\|y_{i}^{0}\right\|_{L^{\infty}(\Omega)} \leq N / 2, i=1,2 .
$$

By (2.7) and (2.8), we deduce that $y_{1}^{N}, y_{2}^{N} \in[-N, N]$, for all $t \in(0, s)$. Thus, for $t \in(0, s)$, $f_{1}^{N}$ and $f_{2}^{N}$ are defined by their first form, that is $f^{N}\left(t, y_{1}, y_{2}\right)=f\left(t, y_{1}, y_{2}\right)$. This means that $y^{N}=\left(y_{1}^{N}, y_{2}^{N}\right)$ is a local solution of $(1.1)-(1.3)$, defined at least on the set $(0, s) \times \Omega$.

We prove now that $\left\|y_{i}\right\|_{L^{\infty}((0, s) \times \Omega)} \leq K, i=1,2$, for some positive constant $K$. Indeed, comparing the solution $y_{1}$ of our problem with the solution of problem

$$
\left\{\begin{array}{c}
\frac{\partial z_{1}}{\partial t}=\alpha_{1} \Delta z_{1}+r z_{1}, \quad(t, x) \in Q \\
\frac{\partial z_{1}}{\partial \nu}=0,(t, x) \in \Sigma \\
z_{1}(0, x)=y_{1}^{0}(x), x \in \Omega
\end{array}\right.
$$

we find that $0<y_{1}(t, x) \leq z_{1}(t, x),(\forall)(t, x) \in(0, s) \times \Omega$. As above, we can easily prove that $z_{1} \in L^{\infty}(Q)$, so $\left\|y_{1}\right\|_{L^{\infty}(Q)} \leq K_{1}$, for some constant $K_{1}>0$. Analogously we can show that $\left\|y_{2}\right\|_{L^{\infty}((0, s) \times \Omega)} \leq K_{2}$, with $K_{2}>0$. This means that $y=\left(y_{1}, y_{2}\right)$ is defined on the whole set $Q$. Moreover, it is positive, bounded on $Q$ and satisfies the conditions $y_{i} \in W^{1,2}\left(0, T ; L^{2}(\Omega)\right) \cap$ $L^{2}\left(0, T ; H^{2}(\Omega)\right), i=1,2$.

In the sequel, we derive some regularity results. Taking the second power of the first equation from (1.1), integrating by parts on $[0, t] \times \Omega$ and using Green's formula, we deduce that

$$
\begin{gathered}
\int_{0}^{t} \int_{\Omega}\left|\frac{\partial y_{1}}{\partial t}\right|^{2} d s d x+\alpha_{1}^{2} \int_{0}^{t} \int_{\Omega}\left|\Delta y_{1}\right|^{2} d s d x+2 \alpha_{1} \int_{\Omega}\left|\nabla y_{1}\right|^{2} d x- \\
-2 \alpha_{1} \int_{\Omega}\left|\nabla y_{1}^{0}\right|^{2} d x=\int_{0}^{t} \int_{\Omega} f_{1}\left(t, y_{1}, y_{2}\right)^{2} d s d x
\end{gathered}
$$

Now we use the estimates

$$
f_{1}\left(t, y_{1}, y_{2}\right)^{2} \leq K_{3},\left\|y_{1}(t)\right\|_{H^{2}(\Omega)} \leq K_{4}\left\|\Delta y_{1}(t)\right\|_{L^{2}(\Omega)}, t \in[0, T]
$$


for some $K_{3}, K_{4}>0$ and conclude that

$$
\left\|\frac{\partial y_{1}}{\partial t}\right\|_{L^{2}\left(0, T: L^{2}(\Omega)\right)}^{2}+\alpha_{1}^{2}\left\|y_{1}\right\|_{L^{2}\left(0, T: H^{2}(\Omega)\right)}^{2}+2 \alpha_{1}\left\|y_{1}(t)\right\|_{H^{1}(\Omega)}^{2} \leq K_{5}
$$

and similarly for $y_{2}$.

Thus we have proved the following result.

Theorem 2. Let $\alpha_{1}, \alpha_{2}, r, k, b, c, d, m>0$ and $y^{0}=\left(y_{1}^{0}, y_{2}^{0}\right) \in H^{2}(\Omega)^{2}$ such that $y_{1}^{0}, y_{2}^{0}>0$ on $\Omega$ and $\frac{\partial y_{1}^{0}}{\partial \nu}=\frac{\partial y_{2}^{0}}{\partial \nu}=0$ on $\partial \Omega$. If $(u, v) \in U$ and $(i)-($ iii $)$ hold, then problem $(1.1)-(1.3)$ has a unique strong solution $y=\left(y_{1}, y_{2}\right) \in W^{1,2}\left(0, T ; L^{2}(\Omega)^{2}\right)$, with $y_{1}, y_{2} \in L^{\infty}(Q)$, $y_{1}>0$, $y_{2}>0$ on $Q$ and $y_{1}, y_{2} \in L^{2}\left(0, T ; H^{2}(\Omega)\right) \cap L^{\infty}\left(0, T ; H^{1}(\Omega)\right)$. In addition, there exists $K>0$ independent of $(u, v)$, such that for a. e. $t \in[0, T]$,

$$
\left\|\frac{\partial y}{\partial t}\right\|_{L^{2}\left(0, T: L^{2}(\Omega)\right)^{2}}+\|y\|_{L^{2}\left(0, T: H^{2}(\Omega)\right)^{2}}+\|y(t)\|_{H^{1}(\Omega)^{2}}+\|y\|_{L^{\infty}(Q)^{2}} \leq K
$$

\section{Existence of the optimal solution}

In this section we prove the existence of an optimal solution.

Theorem 3. Under the hypotheses of the previous section, problem (1.1) - (1.4) admits an optimal solution $\left(y_{1}^{*}, y_{2}^{*}, u^{*}, v^{*}\right)$.

Proof. Let $d=\inf \{J(u, v)\}$ subject to $(1.1)-(1.3)$ and $(u, v) \in U$, where $J(u, v)$ is defined through (1.4). Since $u, v, y_{1}, y_{2} \in L^{\infty}(Q), d$ is finite. Therefore, there exists a sequence $\left(y_{1 n}, y_{2 n}, u_{n}, v_{n}\right)$ of solutions of problem $(1.1)-(1.3)$ with $\left(u_{n}, v_{n}\right) \in U$ instead of $(u, v)$, such that

$$
d \leq J\left(u_{n}, v_{n}\right) \leq d+\frac{1}{n},(\forall) n \geq 1
$$

So $\left(y_{1 n}, y_{2 n}, u_{n}, v_{n}\right)$ verifies the boundary value problem

$$
\begin{gathered}
\frac{\partial y_{1 n}}{\partial t}=\alpha_{1} \Delta y_{1 n}+r y_{1 n}\left(1-\frac{y_{1 n}}{k} v_{n}\right)-u_{n} y_{2 n} F\left(y_{1 n}, y_{2 n}\right) \\
\frac{\partial y_{2 n}}{\partial t}=\alpha_{2} \Delta y_{2 n}+y_{2 n}\left[-d+c u_{n} F\left(y_{1 n}, y_{2 n}\right)\right] \\
\frac{\partial y_{1 n}}{\partial \nu}=\frac{\partial y_{2 n}}{\partial \nu}=0,(t, x) \in \Sigma,
\end{gathered},(t, x) \in Q,
$$


Inequality (2.12) leads to the estimates

$$
\left\|\frac{\partial y_{i n}}{\partial t}\right\|_{L^{2}\left(0, T: L^{2}(\Omega)\right)} \leq C,\left\|y_{i n}(t)\right\|_{H^{1}(\Omega)} \leq C,\left\|y_{i n}\right\|_{L^{2}\left(0, T ; H^{2}(\Omega)\right)} \leq C,
$$

for all $n \geq 1, t \in[0, T]$ and $i=1,2$.

Since $\left(y_{i n}\right)$ is bounded in $C\left([0, T] ; L^{2}(\Omega)\right),\left(\partial y_{i n} / \partial t\right)$ is bounded in $L^{2}\left(0, T ; L^{2}(\Omega)\right)$, and $\left(y_{\text {in }}(t)\right)$ is compact in $L^{2}(\Omega)$, for each $t \in[0, T]$ (because $H^{1}(\Omega)$ is compactly imbedded in $L^{2}(\Omega)$ ), by Ascoli-Arzela Theorem it follows that $\left(y_{\text {in }}\right)$ is compact in $C\left([0, T] ; L^{2}(\Omega)\right)$. Thus, at least on a subsequence denoted again $\left(y_{i n}\right)$, we have

$$
y_{\text {in }} \rightarrow y_{i}^{*} \text { in } L^{2}(\Omega) \text { uniformly with respect to } t, i=1,2 .
$$

Since $\left(\Delta y_{i n}\right)$ is bounded in $L^{2}(Q)$ (in view of $(i i)$ and of the boundedness of $y_{i n}, \partial y_{i n} / \partial t$ ), it is weakly convergent on a subsequence (in the sense of distributions). But for every distribution $\mu$,

$$
\int_{Q} \mu \Delta y_{i n} d t d x=\int_{Q} y_{i n} \Delta \mu d t d x \rightarrow \int_{Q} y_{i}^{*} \Delta \mu d t d x=\int_{Q} \mu \Delta y_{i}^{*} d t d x .
$$

This implies that $\Delta y_{i n} \rightarrow \Delta y_{i}^{*}$ (weakly) in $L^{2}(Q)$. On the other hand, inequalities (3.5) infer that

$$
\begin{gathered}
\frac{\partial y_{i n}}{\partial t} \rightarrow \frac{\partial y_{i}^{*}}{\partial t} \text { in } L^{2}(Q), \\
y_{\text {in }} \rightarrow y_{i}^{*} \text { weakly in } L^{2}\left(0, T ; H^{1}(\Omega)\right), \\
y_{\text {in }} \rightarrow y_{i}^{*} \text { weakly in } L^{2}\left(0, T ; H^{2}(\Omega)\right) .
\end{gathered}
$$

We now show that

$$
y_{2 n} F\left(y_{1 n}, y_{2 n}\right) \rightarrow y_{2}^{*} F\left(y_{1}^{*}, y_{2}^{*}\right) \text { in } L^{2}\left(0, T ; L^{2}(\Omega)\right) \text {. }
$$

Indeed, since $y_{\text {in }} \rightarrow y_{i}^{*}$ (strongly) in $L^{2}\left(0, T ; L^{2}(\Omega)\right),\left(y_{i n}\right)$ is bounded in $L^{\infty}(Q)$, and $F$ is continuous in both variables, from the equality

$$
y_{2 n} F\left(y_{1 n}, y_{2 n}\right)-y_{2}^{*} F\left(y_{1}^{*}, y_{2}^{*}\right)=\left[F\left(y_{1 n}, y_{2 n}\right)-F\left(y_{1}^{*}, y_{2}^{*}\right)\right] y_{2 n}+\left(y_{2 n}-y_{2}^{*}\right) F\left(y_{1}^{*}, y_{2}^{*}\right),
$$

we derive (3.6). Similarly we can show that $y_{1 n}^{2} \rightarrow\left(y_{1}^{*}\right)^{2}$.

On a subsequence denoted again $\left(u_{n}, v_{n}\right)$, we have $u_{n} \rightarrow u^{*}, v_{n} \rightarrow v^{*}$ in $L^{2}(Q)$. Since $U$ is closed and convex, it is also weakly closed, hence $\left(u^{*}, v^{*}\right) \in U$. Then we can easily see that $u_{n} y_{2 n} F\left(y_{1 n}, y_{2 n}\right) \rightarrow u^{*} y_{2}^{*} F\left(y_{1}^{*}, y_{2}^{*}\right)$ and $v_{n} y_{1 n}^{2} \rightarrow v^{*}\left(y_{1}^{*}\right)^{2}$ in $L^{2}\left(0, T ; L^{2}(\Omega)\right)$.

Letting $n$ go to $\infty$ in $(3.1)-(3.4)$, we deduce that $\left(y_{1}^{*}, y_{2}^{*}, u^{*}, v^{*}\right)$ verifies problem $(1.1)-(1.3)$ and minimize the cost functional (1.4). The proof is complete. 


\section{Necessary optimality conditions}

In this section we deduce the optimality system. Let $y^{*}=\left(y_{1}^{*}, y_{2}^{*}\right)$ be the solution of $(1.1)-(1.3)$ corresponding to the optimal control $\left(u^{*}, v^{*}\right)$. The adjoint system to $(2.4)$ is

$$
\left\{\begin{array}{c}
p^{\prime}(t)+A^{*} p(t)=-f_{y}^{*}\left(t, y^{*}\right) p+L_{y} \text { a. e. } t \in(0, T) \\
p(T)=-\nabla \psi\left(y^{*}(T)\right),
\end{array}\right.
$$

where $\psi(y(T, \cdot))=-l_{1} y_{1}(T, \cdot)-l_{2} y_{2}(T, \cdot), L(y)=-k_{1} y_{1}-k_{2} y_{2}, A^{*}$ is the adjoint of the operator $A, p=\left(p_{1}, p_{2}\right)$ is the adjoint variable, and $f_{y}^{*}$ is the adjoint of the Jacobian matrix $f_{y}$. This system can be written as

$$
\left\{\begin{array}{l}
\frac{\partial p_{1}}{\partial t}=-\alpha_{1} \Delta p_{1}-r p_{1}+\frac{2 r v^{*}}{k} y_{1}^{*} p_{1}-k_{1}+u^{*} y_{2}^{*} \frac{\partial F}{\partial y_{1}}\left(y_{1}^{*}, y_{2}^{*}\right)\left(p_{1}-c p_{2}\right) \\
\frac{\partial p_{2}}{\partial t}=-\alpha_{2} \Delta p_{2}+d p_{2}-k_{2}+u^{*}\left(p_{1}-c p_{2}\right)\left[F\left(y_{1}^{*}, y_{2}^{*}\right)+y_{2}^{*} \frac{\partial F}{\partial y_{2}}\left(y_{1}^{*}, y_{2}^{*}\right)\right]
\end{array}\right.
$$

on $Q$, the boundary conditions are

$$
\frac{\partial p_{1}}{\partial \nu}=\frac{\partial p_{2}}{\partial \nu}=0 \text { on } \Sigma=(0, T) \times \partial \Omega
$$

and the transversality conditions are

$$
p_{1}(T, x)=l_{1}, p_{2}(T, x)=l_{2} \text { on } \Omega .
$$

First we prove the existence of the solution to this problem.

Proposition 4. If $\left(y_{1}^{*}, y_{2}^{*}, u^{*}, v^{*}\right)$ is an optimal element of the problem $(1.1)-(1.4)$, then the adjoint system $(4.2)-(4.4)$ has a unique strong solution $p=\left(p_{1}, p_{2}\right) \in W^{1,2}(0, T ; H)$, with $p_{1}$, $p_{2} \in L^{\infty}\left(0, T ; H^{1}(\Omega)\right) \cap L^{2}\left(0, T ; H^{2}(\Omega)\right)$ and $p_{1}, p_{2} \in L^{\infty}(Q)$.

Proof. Let $s=T-t$ and $q=\left(q_{1}, q_{2}\right)$ be given by $q_{i}(s, x)=p_{i}(T-s, x)=p_{i}(t, x),(t, x) \in Q$, $i=1,2$. Problem $(4.2)-(4.4)$ becomes

$$
\left\{\begin{array}{c}
\frac{\partial q_{1}}{\partial s}=\alpha_{1} \Delta q_{1}+r q_{1}-\frac{2 r v^{*}}{k} y_{1}^{*} q_{1}+k_{1}-u^{*} y_{2}^{*} \frac{\partial F}{\partial y_{1}}\left(y_{1}^{*}, y_{2}^{*}\right)\left(q_{1}-c q_{2}\right) \\
\frac{\partial q_{2}}{\partial s}=\alpha_{2} \Delta q_{2}-d q_{2}+k_{2}-u^{*}\left(q_{1}-c q_{2}\right)\left[F\left(y_{1}^{*}, y_{2}^{*}\right)+y_{2}^{*} \frac{\partial F}{\partial y_{2}}\left(y_{1}^{*}, y_{2}^{*}\right)\right]
\end{array}\right.
$$

on $Q$, the boundary conditions are

$$
\frac{\partial q_{1}}{\partial \nu}=\frac{\partial q_{2}}{\partial \nu}=0 \text { on } \Sigma=(0, T) \times \partial \Omega
$$

and the transversality conditions are

$$
q_{1}(0, x)=l_{1}, q_{2}(0, x)=l_{2} \text { on } \Omega .
$$


The claim follows by applying Theorem 1 for this linear problem with bounded coefficients. The proposition is proved.

Let $y^{*}=\left(y_{1}^{*}, y_{2}^{*}\right)$ and $y^{\varepsilon}=\left(y_{1}^{\varepsilon}, y_{2}^{\varepsilon}\right)$ be the solution of $(1.1)-(1.3)$ corresponding to the optimal control $\left(u^{*}, v^{*}\right)$ and to the control $\left(u^{\varepsilon}, v^{\varepsilon}\right), \varepsilon>0$, respectively, where $u^{\varepsilon}=u^{*}+\varepsilon u_{0}, v^{\varepsilon}=v^{*}+\varepsilon v_{0}$, for some $\left(u_{0}, v_{0}\right) \in L^{2}(Q)$ chosen such that $0 \leq u^{*}+\varepsilon u_{0} \leq 1,0<a \leq v^{*}+\varepsilon v_{0} \leq 1$ a. e. on $[0, T]$. Subtracting the system $(1.1)-(1.3)$ corresponding to $\left(u^{*}, v^{*}\right)$ from that corresponding to $\left(u^{\varepsilon}, v^{\varepsilon}\right)$ and denoting $z_{i}^{\varepsilon}=\left(y_{i}^{\varepsilon}-y_{i}^{*}\right) / \varepsilon$, we arrive at

$$
\left\{\begin{array}{c}
\frac{\partial z_{1}^{\varepsilon}}{\partial t}=\alpha_{1} \Delta z_{1}^{\varepsilon}+\left[r-\frac{r}{k} v^{*}\left(y_{1}^{\varepsilon}+y_{1}^{*}\right)-u^{*} y_{2}^{*} \frac{F\left(y_{1}^{\varepsilon}, y_{2}^{\varepsilon}\right)-F\left(y_{1}^{*}, y_{2}^{\varepsilon}\right)}{y_{1}^{\varepsilon}-y_{1}^{*}}\right] z_{1}^{\varepsilon}- \\
-u^{*}\left[F\left(y_{1}^{\varepsilon}, y_{2}^{\varepsilon}\right)+y_{2}^{*} \frac{F\left(y_{1}^{*}, y_{2}^{\varepsilon}\right)-F\left(y_{1}^{*}, y_{2}^{*}\right)}{y_{2}^{\varepsilon}-y_{2}^{*}}\right] z_{2}^{\varepsilon}-\frac{r}{k} v_{0}\left(y_{1}^{\varepsilon}\right)^{2}-u_{0} y_{2}^{\varepsilon} F\left(y_{1}^{\varepsilon}, y_{2}^{\varepsilon}\right) \\
\frac{\partial z_{2}^{\varepsilon}}{\partial t}=\alpha_{2} \Delta z_{2}^{\varepsilon}+c u^{*} y_{2}^{*} \frac{F\left(y_{1}^{\varepsilon}, y_{2}^{\varepsilon}\right)-F\left(y_{1}^{*}, y_{2}^{\varepsilon}\right)}{y_{1}^{\varepsilon}-y_{1}^{*}} z_{1}^{\varepsilon}+\left[-d+c u^{*} F\left(y_{1}^{\varepsilon}, y_{2}^{\varepsilon}\right)+\right. \\
\left.+c u^{*} y_{2}^{*} \frac{F\left(y_{1}^{*}, y_{2}^{\varepsilon}\right)-F\left(y_{1}^{*}, y_{2}^{*}\right)}{y_{2}^{\varepsilon}-y_{2}^{*}}\right] z_{2}^{\varepsilon}+c u_{0} y_{2}^{\varepsilon} F\left(y_{1}^{\varepsilon}, y_{2}^{\varepsilon}\right)
\end{array}\right.
$$

for $(t, x) \in Q$,

$$
\begin{gathered}
\frac{\partial z_{1}^{\varepsilon}}{\partial \nu}=\frac{\partial z_{2}^{\varepsilon}}{\partial \nu}=0,(t, x) \in \Sigma, \\
z_{1}^{\varepsilon}(0, x)=z_{2}^{\varepsilon}(0, x)=0, x \in \Omega .
\end{gathered}
$$

As in Proposition 4, we can easily see that this problem admits a unique strong solution $\left(z_{1}^{\varepsilon}, z_{2}^{\varepsilon}\right)$ which is bounded on $Q$.

Proposition 5. There exist the limits $z_{i}=\lim _{\varepsilon \rightarrow 0} \frac{y_{i}^{\varepsilon}-y_{i}^{*}}{\varepsilon}$ in $L^{2}(Q), i=1,2$. In addition, they satisfy the boundary value problem

$$
\left\{\begin{array}{c}
\frac{\partial z_{1}}{\partial t}=\alpha_{1} \Delta z_{1}+\left[r-\frac{2 r}{k} v^{*} y_{1}^{*}-u^{*} y_{2}^{*} \frac{\partial F}{\partial y_{1}}\left(y_{1}^{*}, y_{2}^{*}\right)\right] z_{1}- \\
-u^{*}\left[F\left(y_{1}^{*}, y_{2}^{*}\right)+y_{2}^{*} \frac{\partial F}{\partial y_{2}}\left(y_{1}^{*}, y_{2}^{*}\right)\right] z_{2}-\frac{r}{k} v_{0}\left(y_{1}^{*}\right)^{2}-u_{0} y_{2}^{*} F\left(y_{1}^{*}, y_{2}^{*}\right) \\
\frac{\partial z_{2}}{\partial t}=\alpha_{2} \Delta z_{2}+c u^{*} y_{2}^{*} \frac{\partial F}{\partial y_{1}}\left(y_{1}^{*}, y_{2}^{*}\right) z_{1}+\left[-d+c u^{*} F\left(y_{1}^{*}, y_{2}^{*}\right)+\right. \\
\left.+c u^{*} y_{2}^{*} \frac{\partial F}{\partial y_{2}}\left(y_{1}^{*}, y_{2}^{*}\right)\right] z_{2}+c u_{0} y_{2}^{*} F\left(y_{1}^{*}, y_{2}^{*}\right) \\
\frac{\partial z_{1}}{\partial \nu}=\frac{\partial z_{2}}{\partial \nu}=0,(t, x) \in \Sigma \\
z_{1}(0, x)=z_{2}(0, x)=0, x \in \Omega .
\end{array}\right.
$$

In addition, $z_{1}, z_{2} \in L^{2}\left(0, T ; H^{2}(\Omega)\right) \cap L^{\infty}\left(0, T ; H^{1}(\Omega)\right)$ and $z_{1}, z_{2} \in L^{\infty}(Q)$. 
Proof. Let $A$ be the operator defined by $(2.1)-(2.2), Z^{\varepsilon}=\left(z_{1}^{\varepsilon}, z_{2}^{\varepsilon}\right)^{T}$,

$$
M^{\varepsilon}=\left(\begin{array}{cc}
M_{11} & M_{12} \\
M_{21} & M_{22}
\end{array}\right), N^{\varepsilon}=\left(\begin{array}{c}
-\frac{r}{k} v_{0}\left(y_{1}^{\varepsilon}\right)^{2}-u_{0} y_{2}^{\varepsilon} F\left(y_{1}^{\varepsilon}, y_{2}^{\varepsilon}\right) \\
c u_{0} y_{2}^{\varepsilon} F\left(y_{1}^{\varepsilon}, y_{2}^{\varepsilon}\right)
\end{array}\right)
$$

where

$$
\left\{\begin{array}{c}
M_{11}=r-\frac{r}{k} v^{*}\left(y_{1}^{\varepsilon}+y_{1}^{*}\right)-u^{*} y_{2}^{*} \frac{F\left(y_{1}^{\varepsilon}, y_{2}^{\varepsilon}\right)-F\left(y_{1}^{*}, y_{2}^{\varepsilon}\right)}{y_{1}^{\varepsilon}-y_{1}^{*}} \\
M_{12}=-u^{*} F\left(y_{1}^{\varepsilon}, y_{2}^{\varepsilon}\right)-u^{*} y_{2}^{*} \frac{F\left(y_{1}^{*}, y_{2}^{\varepsilon}\right)-F\left(y_{1}^{*}, y_{2}^{*}\right)}{y_{2}^{\varepsilon}-y_{2}^{*}} \\
M_{21}=c u^{*} y_{2}^{*} \frac{F\left(y_{1}^{\varepsilon}, y_{2}^{\varepsilon}\right)-F\left(y_{1}^{*}, y_{2}^{\varepsilon}\right)}{y_{1}^{\varepsilon}-y_{1}^{*}} \\
M_{22}=-d+c u^{*} F\left(y_{1}^{\varepsilon}, y_{2}^{\varepsilon}\right)+c u^{*} y_{2}^{*} \frac{F\left(y_{1}^{*}, y_{2}^{\varepsilon}\right)-F\left(y_{1}^{*}, y_{2}^{*}\right)}{y_{2}^{\varepsilon}-y_{2}^{*}}
\end{array}\right.
$$

Then problem $(4.8)-(4.10)$ can be written as an abstract Cauchy problem of the form

$$
\frac{\partial Z^{\varepsilon}}{\partial t}=A\left(Z^{\varepsilon}\right)+M^{\epsilon} Z^{\varepsilon}+N^{\varepsilon}, t \in(0, T), Z^{\varepsilon}(0)=0 .
$$

By a comparison theorem, observe that $0 \leq y_{1}^{\varepsilon}(t, x) \leq Y_{1}(t, x), 0 \leq y_{2}^{\varepsilon}(t, x) \leq Y_{2}(t, x)$, $(\forall)(t, x) \in Q$, where $Y_{1}$ and $Y_{2}$ verify the problems

$$
\left\{\begin{array}{c}
\frac{\partial Y_{1}}{\partial t}=\alpha_{1} \Delta Y_{1}+r Y_{1},(t, x) \in Q \\
\frac{\partial Y_{1}}{\partial \nu}=0,(t, x) \in \Sigma \\
Y_{1}(0, x)=y_{1}^{0}(x), x \in \Omega
\end{array}\right.
$$

and

$$
\left\{\begin{array}{c}
\frac{\partial Y_{2}}{\partial t}=\alpha_{2} \Delta Y_{2}+c m_{1} Y_{2},(t, x) \in Q \\
\frac{\partial Y_{2}}{\partial \nu}=0,(t, x) \in \Sigma \\
Y_{2}(0, x)=y_{2}^{0}(x), x \in \Omega
\end{array},\right.
$$

respectively. (Here $m_{1}>0$ is an upper bound for $F\left(y_{1}, y_{2}\right)$.) This means that $y_{1}^{\varepsilon}$ and $y_{2}^{\varepsilon}$ are bounded on $Q$, uniformly with respect to $\varepsilon$. Thus $M^{\varepsilon}$ and $N^{\varepsilon}$ have also bounded elements in $Q$, uniformly with respect to $\varepsilon$.

If $\{S(t), t \geq 0\}$ is the $C_{0}$-semigroup generated by $A$, since $S(t)(0)=0$, the solution of (4.14) can be expressed as

$$
Z^{\varepsilon}(t)=\int_{0}^{t} S(t-s) M^{\epsilon}(s) Z^{\varepsilon}(s) d s+\int_{0}^{t} S(t-s) N^{\varepsilon}(s) d s .
$$

In view of the boundedness of $M^{\varepsilon}$ and $N^{\varepsilon}$, uniformly with respect to $\varepsilon$, by Gronwall's inequality, one obtains $\|\left. Z^{\varepsilon}\right|_{L^{2}(Q)^{2}} \leq m_{2}$, for some constant $m_{2}>0$. Thus, we have proved that $z_{1}^{\varepsilon}$ and $z_{2}^{\varepsilon}$ 
are bounded in $L^{2}(Q)$, uniformly with respect to $\varepsilon$. Since $\left\|y_{i}^{\varepsilon}-y_{i}^{*}\right\|_{L^{2}(Q)}=\varepsilon\left\|z_{i}^{e}\right\|_{L^{2}(Q)} \rightarrow 0$ as $\varepsilon \rightarrow 0$, one gets $y_{i}^{\varepsilon} \rightarrow y_{i}^{*}($ as $\varepsilon \rightarrow 0)$ in $L^{2}(Q)$, for $i=1,2$.

Consider now the system obtained by passing to the limit formally as $\varepsilon \rightarrow 0$ in (4.8) $-(4.10)$, that is the system $(4.11)-(4.13)$. If we denote $Z=\left(z_{1}, z_{2}\right)^{T}$, this problem can be written as

$$
\frac{\partial Z}{\partial t}=A(Z)+M Z+N, t \in(0, T), Z(0)=0,
$$

where

$$
M=\left(\begin{array}{cc}
r-\frac{2 r}{k} v^{*} y_{1}^{*}-u^{*} y_{2}^{*} \frac{\partial F\left(y_{1}^{*}, y_{2}^{*}\right)}{\partial y_{1}} & -u^{*} F\left(y_{1}^{*}, y_{2}^{*}\right)-u^{*} y_{2}^{*} \frac{\partial F\left(y_{1}^{*}, y_{2}^{*}\right)}{\partial y_{2}} \\
c u^{*} y_{2}^{*} \frac{\partial F\left(y_{1}^{*}, y_{2}^{*}\right)}{\partial y_{1}} & -d+c u^{*} F\left(y_{1}^{*}, y_{2}^{*}\right)+c u^{*} y_{2}^{*} \frac{\partial F\left(y_{1}^{*}, y_{2}^{*}\right)}{\partial y_{2}}
\end{array}\right)
$$

and

$$
N=\left(\begin{array}{c}
-\frac{r}{k} v_{0}\left(y_{1}^{*}\right)^{2}-u_{0} y_{2}^{*} F\left(y_{1}^{*}, y_{2}^{*}\right) \\
c u_{0} y_{2}^{*} F\left(y_{1}^{*}, y_{2}^{*}\right)
\end{array}\right)
$$

Then its solution can be expressed in the form

$$
Z(t)=\int_{0}^{t} S(t-s) M(s) Z(s) d s+\int_{0}^{t} S(t-s) N(s) d s .
$$

Subtracting (4.16) from (4.15), we get

$$
\begin{aligned}
Z^{\varepsilon}(t)-Z(t) & =\int_{0}^{t} S(t-s) M^{\varepsilon}(s)\left[Z^{\varepsilon}(s)-Z(s)\right] d s+ \\
& +\int_{0}^{t} S(t-s)\left\{N^{\varepsilon}(s)-N(s)+\left[M^{\varepsilon}(s)-M(s)\right] Z(s)\right\} d s .
\end{aligned}
$$

Since $y_{i}^{\varepsilon} \rightarrow y_{i}^{*}$ in $L^{2}(Q)$ and $F \in C^{1}\left((0, \infty)^{2}\right)$, it follows that all the elements of the matrix $M^{\varepsilon}$ tend to the elements of $M$ in $L^{2}(Q)$ (as $\varepsilon \rightarrow 0$ ) and similarly for $N^{\varepsilon}$ and $N$. Using this, together with Gronwall's inequality, we arrive at the conclusion that $z_{1}^{\varepsilon} \rightarrow z_{1}, z_{2}^{\varepsilon} \rightarrow z_{2}$ in $L^{2}(Q)$, as $\varepsilon \rightarrow 0$. This completes the proof.

Now we can state the main result of this section.

Theorem 6. If $\left(y_{1}^{*}, y_{2}^{*}, u^{*}, v^{*}\right)$ is an optimal element for problem (1.1) - (1.4), then there exists $p=\left(p_{1}, p_{2}\right) \in W^{1,2}(0, T ; H)$, such that the following optimality system is verified:

$$
\left\{\begin{array}{c}
\frac{\partial y_{1}^{*}}{\partial t}=\alpha_{1} \Delta y_{1}^{*}+r y_{1}^{*}\left(1-\frac{y_{1}^{*}}{k} v^{*}\right)-u^{*} y_{2}^{*} F\left(y_{1}^{*}, y_{2}^{*}\right) \\
\frac{\partial y_{2}^{*}}{\partial t}=\alpha_{2} \Delta y_{2}^{*}+y_{2}^{*}\left[-d+c u^{*} F\left(y_{1}^{*}, y_{2}^{*}\right)\right] \\
\frac{\partial y_{1}^{*}}{\partial \nu}=\frac{\partial y_{2}^{*}}{\partial \nu}=0,(t, x) \in \Sigma,
\end{array},(t, x) \in Q\right.
$$




$$
\begin{gathered}
y_{1}^{*}(0, x)=y_{1}^{0}(x), y_{2}^{*}(0, x)=y_{2}^{0}(x), x \in \Omega \\
\left\{\begin{array}{c}
\frac{\partial p_{1}}{\partial t}=-\alpha_{1} \Delta p_{1}-r p_{1}+\frac{2 r v^{*}}{k} y_{1}^{*} p_{1}-k_{1}+u^{*} y_{2}^{*} \frac{\partial F}{\partial y_{1}}\left(y_{1}^{*}, y_{2}^{*}\right)\left(p_{1}-c p_{2}\right) \\
\frac{\partial p_{2}}{\partial t}=-\alpha_{2} \Delta p_{2}+d p_{2}-k_{2}+u^{*}\left(p_{1}-c p_{2}\right)\left[F\left(y_{1}^{*}, y_{2}^{*}\right)+y_{2}^{*} \frac{\partial F}{\partial y_{2}}\left(y_{1}^{*}, y_{2}^{*}\right)\right] \\
\frac{\partial p_{1}}{\partial \nu}=\frac{\partial p_{2}}{\partial \nu}=0 \text { on } \Sigma \\
p_{1}(T, x)=l_{1}, p_{2}(T, x)=l_{2} \text { on } \Omega, \\
u^{*}(t, x)=\left\{\begin{array}{c}
0, p_{1}-c p_{2}>0 \\
1, p_{1}-c p_{2}<0
\end{array}, v^{*}(t, x)=\left\{\begin{array}{l}
a, p_{1}>0 \\
1, p_{1}<0
\end{array}\right.\right.
\end{array} .\right.
\end{gathered}
$$

Proof. We take $u^{\varepsilon}=u^{*}+\varepsilon u_{0}, v^{\varepsilon}=v^{*}+\varepsilon v_{0}$, with $\left(u_{0}, v_{0}\right) \in L^{2}(Q)^{2}$ such that $0 \leq u^{*}+\varepsilon u_{0} \leq 1$, $0<a \leq v^{*}+\varepsilon v_{0} \leq 1$ a. e. on $[0, T]$. As above, let $y^{*}=\left(y_{1}^{*}, y_{2}^{*}\right)$ and $y^{\varepsilon}=\left(y_{1}^{\varepsilon}, y_{2}^{\varepsilon}\right)$ be the solutions of $(1.1)-(1.3)$ corresponding to $\left(u^{*}, v^{*}\right)$ and to $\left(u^{\varepsilon}, v^{\varepsilon}\right), \varepsilon>0$, respectively. Then

$$
J\left(u^{*}, v^{*}\right) \leq J\left(u^{\varepsilon}, v^{\varepsilon}\right),(\forall) \varepsilon>0 .
$$

Dividing by $\varepsilon>0$ and passing to the limit as $\varepsilon \rightarrow 0$, we arrive at

$$
\int_{\Omega}\left[l_{1} z_{1}(T, x)+l_{2} z_{2}(T, x)\right] d x+\int_{Q}\left(k_{1} z_{1}+k_{2} z_{2}\right)(t, x) d t d x \leq 0 .
$$

Multiplying the equations of (4.11) by $p_{1}$ and $p_{2}$, respectively and the equations of (4.20) by $z_{1}$ and $z_{2}$, respectively, one obtains

$$
\begin{gathered}
\left(p_{1} \frac{\partial z_{1}}{\partial t}+z_{1} \frac{\partial p_{1}}{\partial t}\right)+\left(p_{2} \frac{\partial z_{2}}{\partial t}+z_{2} \frac{\partial p_{2}}{\partial t}\right)=\alpha_{1}\left(p_{1} \Delta z_{1}-z_{1} \Delta p_{1}\right)+\alpha_{2}\left(p_{2} \Delta z_{2}-z_{2} \Delta p_{2}\right)- \\
-\frac{r}{k} v_{0} p_{1}\left(y_{1}^{*}\right)^{2}+u_{0} y_{2}^{*} F\left(y_{1}^{*}, y_{2}^{*}\right)\left(c p_{2}-p_{1}\right)-\left(k_{1} z_{1}+k_{2} z_{2}\right) .
\end{gathered}
$$

One integrates over $Q$. In view of Green's formula and of $(4.12),(4.13),(4.21),(4.22)$, one deduces that

$$
\begin{aligned}
& \int_{\Omega}\left(l_{1} z_{1}+l_{2} z_{2}\right)(T, x) d x+\int_{Q}\left(k_{1} z_{1}+k_{2} z_{2}\right)(t, x) d t d x= \\
& =-\frac{r}{k} \int_{Q} v_{0} p_{1}\left(y_{1}^{*}\right)^{2} d t d x+\int_{Q} u_{0} y_{2}^{*} F\left(y_{1}^{*}, y_{2}^{*}\right)\left(c p_{2}-p_{1}\right) .
\end{aligned}
$$

By (4.24), we get

$$
-\frac{r}{k} \int_{Q} v_{0} p_{1}\left(y_{1}^{*}\right)^{2} d t d x+\int_{Q} u_{0} y_{2}^{*} F\left(y_{1}^{*}, y_{2}^{*}\right)\left(c p_{2}-p_{1}\right) \leq 0 .
$$


Taking $u_{0}, v_{0}$ in the form $u_{0}=-u^{*}+\widetilde{u}_{0}, v_{0}=-v^{*}+\widetilde{v}_{0}$ with arbitrary $\left(\widetilde{u}_{0}, \widetilde{v}_{0}\right) \in U$, it follows that

$$
<-u^{*}+\widetilde{u}_{0}, w_{1}>_{L^{2}(Q)}+<-v^{*}+\widetilde{v}_{0}, w_{2}>_{L^{2}(Q)} \geq 0
$$

where $<\cdot, \cdot>_{L^{2}(Q)}$ denotes the scalar product in $L^{2}(Q)$ and

$$
w_{1}(t, x)=y_{2}^{*} F\left(y_{1}^{*}, y_{2}^{*}\right)\left(p_{1}-c p_{2}\right), w_{2}(t, x)=\frac{r}{k} p_{1}\left(y_{1}^{*}\right)^{2} .
$$

Since $\left(\widetilde{u}_{0}, \widetilde{v}_{0}\right)$ is arbitrary from $U$, in view of hypothesis $(i i i)$, this implies $(4.23)$. The theorem is proved.

Remark 7. Observe that $u^{*}(t, x)\left(p_{1}-c p_{2}\right)(t, x) \leq 0$ on $Q$.

We now show that $v^{*}=a$ on $Q$ and find the form of $u^{*}$ in some particular cases, according to the sign of a specific constant.

Proposition 8. Under hypotheses $(i)-($ iii $)$, we have $p_{1}>0$ on $Q$ and thus $v^{*}(t, x)=a$ on $Q$.

Proof. For $s=T-t$, let $q_{i}(s, x)=p_{i}(T-s, x)=p_{i}(t, x),(t, x) \in Q, i=1,2$. The adjoint system can be written in the form (4.5) - (4.7).Comparing the solution $\left(q_{1}, q_{2}\right)$ of this problem with the solution of a similar problem, but with $k_{1}=k_{2}=0, l_{1}=l_{2}=0$, using Remark 4.4, we derive that $q_{1}(t, x) \geq 0, q_{2}(t, x) \geq 0$ on $Q$, so $p_{1} \geq 0, p_{2} \geq 0$ on $Q$.

If $k_{1}>0$ or $l_{1}>0$, then $q_{1}>0$ on $Q$, so $p_{1}>0$ and $v^{*}(t, x)=a$ on $Q$. If $k_{1}=0, l_{1}=0$, then $q_{1}$ can be zero (and consequently $p_{1}=0$ ) if and only if $u^{*}=0$. But this gives a contradiction with (4.23). Thus the only possibility is $p_{1}>0$ on $Q$. This completes the proof.

To study the form of $u^{*}$, we take $\alpha_{1}=\alpha_{2}=\alpha$. Then the switching function $P=p_{1}-c p_{2}$ verifies the following boundary value problem:

$$
\left\{\begin{array}{c}
\frac{\partial P}{\partial t}=-\alpha \Delta P+P\left[d+u^{*}\left(y_{2}^{*} \frac{\partial F}{\partial y_{1}}-c F-c y_{2}^{*} \frac{\partial F}{\partial y_{2}}\right)\left(y_{1}^{*}, y_{2}^{*}\right)\right]- \\
-\left(d+r-\frac{2 r a}{k} y_{1}^{*}\right) p_{1}-\left(k_{1}-c k_{2}\right),(t, x) \in Q \\
\frac{\partial P}{\partial \nu}=0,(t, x) \in \Sigma \\
P(T, x)=l_{1}-c l_{2}, x \in \Omega
\end{array}\right.
$$

As before, making the change of variable $s=T-t$, function $R(s, x)=P(T-s, x)=$ $P(t, x)$ verifies the problem

$$
\left\{\begin{array}{c}
\frac{\partial R}{\partial s}=\alpha \Delta R-R\left[d+u^{*}\left(y_{2}^{*} \frac{\partial F}{\partial y_{1}}-c F-c y_{2}^{*} \frac{\partial F}{\partial y_{2}}\right)\left(y_{1}^{*}, y_{2}^{*}\right)\right]+ \\
+\left(d+r-\frac{2 r a}{k} y_{1}^{*}\right) p_{1}+\left(k_{1}-c k_{2}\right),(t, x) \in Q \\
\frac{\partial R}{\partial \nu}=0,(t, x) \in \Sigma \\
R(0, x)=l_{1}-c l_{2}, x \in \Omega .
\end{array}\right.
$$


Theorem 9. Suppose that hypotheses $(i)-($ iii $)$ hold, the diffusion coefficients coincides $\left(\alpha_{1}=\right.$ $\left.\alpha_{2}=\alpha\right)$ and

$$
k_{1}-c k_{2}>0, d+r-\frac{2 r a}{k} y_{1}^{\max }>0, \text { where } y_{1}^{\max }=\sup _{(t, x) \in Q} y_{1}^{*}(t, x) .
$$

If $\left(u^{*}, v^{*}\right)$ is the optimal control of our problem, then $v^{*}=a$ on $Q$ and for $u^{*}$ we have the following situations:

Case 1. If $l_{1}-c l_{2} \geq 0$, then $u^{*}(t, x)=0$ on $Q$.

Case 2. If $l_{1}-c l_{2}<0$, then for every $x \in \Omega$, there exists $\tau_{x} \in[0, T]$, such that $u^{*}(t, x)=1$, for all $t \in\left(\tau_{x}, T\right]$.

Proof. Case 1. Suppose first that $l_{1}-c l_{2}>0$. Then, $P(T, x)=l_{1}-c l_{2}>0,(\forall) x \in \Omega$. For every $x \in \Omega$, there exists an element $\tau_{x} \in[0, T)$ such that $P(t, x)>0,(\forall) t \in\left(\tau_{x}, T\right]$. Then $u^{*}(t, x)=0,(\forall) t \in\left(\tau_{x}, T\right]$.

Since $l_{1}-c l_{2}>0$ and $p_{1}>0$ on $Q$, it follows by (4.27) and (4.28), with the aid of a comparison result, that $R(s, x) \geq 0$. So $P(t, x) \geq 0,(\forall)(t, x) \in Q$. Moreover, $P(t, x)>0$ on $Q$, so $u^{*}(t, x)=0,(\forall)(t, x) \in Q$.

Case 2. Assume that $l_{1}-c l_{2}=0$. Then, $P(T, x)=l_{1}-c l_{2}=0,(\forall) x \in \Omega$ and

$$
(\partial P / \partial t)(T, x)=-\left(d+r-\frac{2 r a}{k} y_{1}^{*}(T, x)\right) l_{1}-\left(k_{1}-c k_{2}\right)<0,(\forall) x \in \Omega .
$$

Therefore $P(t, x)>0$ in a left neighborhood $\left(\tau_{x}, T\right)$ of $T$. We compare again the solution of (4.27) with the solution of a similar problem, with $R(0, x)=0$ and omitting the terms

$$
\left(d+r-\frac{2 r a}{k} y_{1}^{*}\right) p_{1}(T-s, x) \text { and } k_{1}-c k_{2} .
$$

It follows that $R(s, x)>0$ and consequently, $u^{*}(t, x)=0,(\forall)(t, x) \in Q$.

Case 3. Let $l_{1}-c l_{2}<0$. Then, $P(T, x)=l_{1}-c l_{2}<0,(\forall) x \in \Omega$. For each $x \in \Omega$, there exists a neighborhood $\left(\tau_{x}, T\right]$ of $T$, such that $P(t, x)<0,(\forall) t \in\left(\tau_{x}, T\right]$. Here $u^{*}(t, x)=1$, $(\forall) t \in\left(\tau_{x}, T\right]$. The theorem is proved.

Remark 10. Remark that, in order to minimize the cost functional, the prey individuals should have the smallest degree of mixture on the whole domain $Q$ (their rate of mixture is $v^{*}=a$ on the $Q)$. If $u^{*}(t, x)=0$, this means that at time $t$ and point $x \in \Omega$, the two species should be completely separated. If $u^{*}(t, x)=1$, then the prey and predator are completely mixed. In this situation, the ecosystem would not be controlled at all. The differential system coincides with the initial one, missing $u$ and $v$. 


\section{Acknowledgements}

This work was supported by CNCSIS- UEFISCSU project number PN II -IDEI 381/2007, Romania, "Optimal control and stabilization of the continuous models of population dynamics".

\section{References}

[1] N. Apreutesei. Necessary optimality conditions for a Lotka-Volterra three species system. Math. Model. Nat. Phen., 1 (2006), 123-135.

[2] N. Apreutesei. An optimal control problem for prey-predator system with a general functional response. Appl. Math. Letters, 22 (2009), no. 7, 1062-1065.

[3] V. Barbu. Mathematical methods in optimization of differential systems. Kluwer Academic Publishers, Dordrecht, 1994.

[4] G. Feichtinger, G. Tragler, V. Veliov. Optimality conditions for age-structured control systems. J. Math. Anal. Appl., 288 (2003), no. 1, 47-68.

[5] M. Garvie, C. Trenchea. Optimal control of a nutrient-phytoplankton-zooplankton-fish system. SIAM J. Control Optim., 46 (2007), no. 3, 775-791.

[6] Z. He, S. Hong, C. Zhang. Double control problems of age-distributed population dynamics. Nonlinear Anal., Real World Appl., 10 (2009), no. 5, 3112-3121.

[7] I. Hrinca, An optimal control problem for the Lotka-Volterra system with diffusion. Panam. Math. J., 12 (2002), no. 3, 23-46.

[8] N. Kato. Maximum principle for optimal harvesting in linear size-structured population. Math. Popul. Stud., 15 (2008), no. 2, 123-136.

[9] Y. Kuang. Some mechanistically derived population models. Math. Biosci. Eng., 4 (2007), no. 4, 1-11.

[10] J. D. Murray. Mathematical Biology. Springer Verlag, Berlin-Heidelberg-New York, third edition, 2002.

[11] A. Pazy. Semigroups of Linear Operators and Applications to Partial Differential Equations. Applied Mathematical Sciences, 44. New York etc., Springer- Verlag, 1983.

[12] S. Xu. Existence of global solutions for a predator-prey model with cross-diffusion. Electron. J. Diff. Eqns., (2008), 1-14.

[13] S. Yosida. Optimal control of prey-predator systems with Lagrange type and Bolza type cost functionals. Proc. Faculty Science Tokai Univ., 18 (1983), 103-118. 